\title{
Real-Space Observation of Quantum Tunneling by a Carbon Atom: Flipping Reaction of Formaldehyde on $\mathrm{Cu}(110)$
}

\author{
Chenfang Lin, ${ }^{\dagger}$ Emile Durant, ${ }^{\ddagger}$ Mats Persson, ${ }^{\dagger}$ Mariana Rossi, ${ }^{\S}{ }^{\circledR}$ and Takashi Kumagai ${ }^{*}, \|_{\odot}$ \\ ${ }^{\dagger}$ Department of Physical Chemistry, Fritz-Haber Institute of the Max-Planck Society, Faradayweg 4-6, 14195 Berlin, Germany \\ ${ }^{\ddagger}$ Surface Science Research Centre and Department of Chemistry, University of Liverpool, Liverpool L69 3BX, U.K.

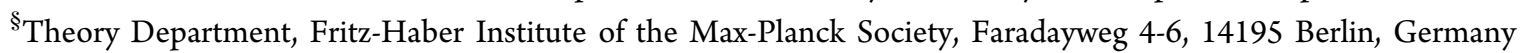 \\ "JST-PRESTO, 4-1-8 Honcho, Kawaguchi, Saitama 332-0012, Japan
}

Supporting Information

ABSTRACT: We present a direct observation of carbon-atom tunneling in the flipping reaction of formaldehyde between its two mirror-reflected states on a $\mathrm{Cu}(110)$ surface using low-temperature scanning tunneling microscopy (STM). The flipping reaction was monitored in real time, and the reaction rate was found to be temperature independent below $10 \mathrm{~K}$. This indicates that this reaction is governed by quantum mechanical tunneling, albeit involving a substantial motion of the carbon atom $(\sim 1 \AA)$. In addition, deuteration of the formaldehyde molecule resulted in a significant kinetic isotope effect $\left(R_{\mathrm{CH}_{2} \mathrm{O}} / R_{\mathrm{CD}_{2} \mathrm{O}} \approx 10\right)$. The adsorption structure, reaction pathway, and tunneling probability were examined by density functional theory calculations, which corroborate the experimental observations.

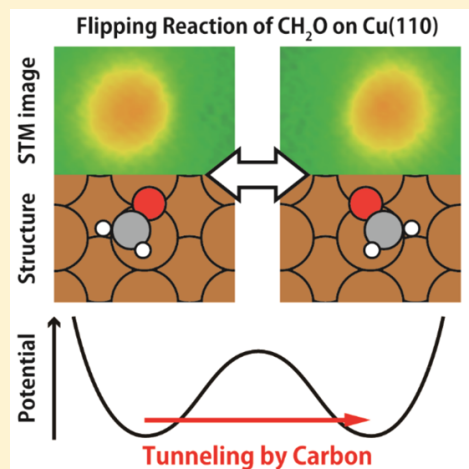

$\mathrm{T}$ unneling is a pure quantum-mechanical effect resulting from the wavelike property of a particle, which makes it possible to pass through a classically insurmountable barrier. Hydrogen tunneling was recognized already in the early days of quantum chemistry ${ }^{1}$ and plays a crucial role in chemical and biological reactions. ${ }^{2-5}$ The contribution of tunneling in chemical reactions strongly depends on the particle mass and decreases rapidly with the increasing mass of the atom. Therefore, heavy-atom tunneling such as carbon and oxygen had been ignored for a long time. However, experimental evidence of carbon tunneling was reported for the tautomerization of cyclobutadiene in the late 20 th century. ${ }^{6-8}$ Since then, several different reactions involving carbon tunneling have been found, ${ }^{9-14}$ even at elevated reaction temperatures. ${ }^{15,16}$ These findings suggest carbon tunneling may be broadly involved in organic reactions.

Tunneling in chemical reactions has been conventionally investigated by spectroscopic methods such as nuclear magnetic resonance and vibrational-rotational spectroscopy. In experiments, the mechanism of carbon tunneling is elusive compared to hydrogen. In the latter case, deuteration of molecules can provide solid evidence through the significant kinetic isotope effects (KIEs), which is rather subtle for ${ }^{12} \mathrm{C} /{ }^{13} \mathrm{C}$, as well as through the temperature dependence of a reaction rate. In the last two decades, low-temperature scanning tunneling microscopy (STM) has emerged as a unique tool to investigate reactions occurring via tunneling. Hydrogen tunneling was examined in diffusion of a hydrogen atom, ${ }^{17}$ in hydrogen-bond exchange reactions in small water clusters, $^{18,19}$ and in single-molecule tautomerization. ${ }^{20,21}$ Remarkably, heavy-atom tunneling was observed for $\mathrm{CO},{ }^{22}$ $\mathrm{Cu}^{23} \mathrm{Co},{ }^{24}$ and cyclooctadiene. ${ }^{25}$ These studies show the capability of STM not only to visualize tunneling dynamics in real space but also to perturb it through a modification of the potential energy surface using the STM tip. ${ }^{20,26}$ So far, the example of heavy-atom tunneling is scarce and a quantitative description remains a challenging task in theoretical simulations. Here we report a new type of reaction involving carbon tunneling, namely, flipping of a formaldehyde molecule on a $\mathrm{Cu}(110)$ surface.

Experiments were carried out under ultrahigh-vacuum conditions $\left(<2 \times 10^{-10}\right.$ mbar $)$ with a low-temperature scanning tunneling microscope from Omicron Nanotechnology $\mathrm{GmbH}$ and operated with a Nanonis controller system. An electrochemically etched tungsten tip was used as a STM probe. The bias voltage $\left(V_{\text {bias }}\right)$ was applied to the sample, and the tip was grounded. The tunneling current $\left(I_{t}\right)$ was collected from the tip. A single-crystalline $\mathrm{Cu}(110)$ surface was cleaned by repeated cycles of argon ion sputtering and annealing to 700 K. Formaldehyde molecules were obtained by sequential dehydrogenation of methanol molecules on the $\mathrm{Cu}(110)$ surface. The clean surface was first exposed to $\mathrm{CH}_{3} \mathrm{OH}$ or $\mathrm{CD}_{3} \mathrm{OD}$ (Aldrich 99\%) gas at $10 \mathrm{~K}$. Adsorbed methanol molecules were then dehydrogenated by applying a voltage

Received: December 20, 2018

Accepted: January 24, 2019

Published: January 24, 2019 
pulse of $0.7-1.0 \mathrm{~V}$ to yield formaldehyde, as described in ref 27.

The equilibrium structures and the minimum energy paths (MEPs) of the adsorbed formaldehyde on the $\mathrm{Cu}(110)$ surface were obtained from periodic, plane-wave density functional theory (DFT) calculations using the Vienna $a b$ initio simulation program (VASP). ${ }^{28}$ The electron-ion core interactions and the exchange-correlation effects were treated using the Projector Augmented Wave (PAW) method ${ }^{29}$ and the vdW-DF-cx version of the van der Waals density functional, ${ }^{30-32}$ respectively. The $\mathrm{Cu}(110)$ surface was represented in a supercell by a five-layer slab with a $2 \times 3$ and $4 \times 6$ surface unit cells and a $25 \AA$ vacuum region. The MEPs were computed using the nudged elastic band method. ${ }^{33,34}$ Further details of the calculations can be found in the Supporting Information.

Parts $a-c$ of Figure 1 display topographic STM images of three different products yielded by dissociation of methoxy on $\mathrm{Cu}(110)$ at $4.5 \mathrm{~K}$, which could be assigned to formaldehyde: an oval protrusion $\left(\mathrm{O}_{\mathrm{pa}}\right.$ : Figure 1a), a pair of protrusion and depression $\left(\mathrm{P}_{\mathrm{pd}}\right.$ : Figure $\left.1 \mathrm{~b}\right)$, and an oval protrusion but with a lower apparent height $\left(\mathrm{O}_{\mathrm{pb}}\right.$ : Figure 1c). The appearance of $\mathrm{O}_{\mathrm{pa}}$ and $\mathrm{O}_{\mathrm{pb}}$ looks similar, but $\mathrm{O}_{\mathrm{pb}}$ flips between two mirror-

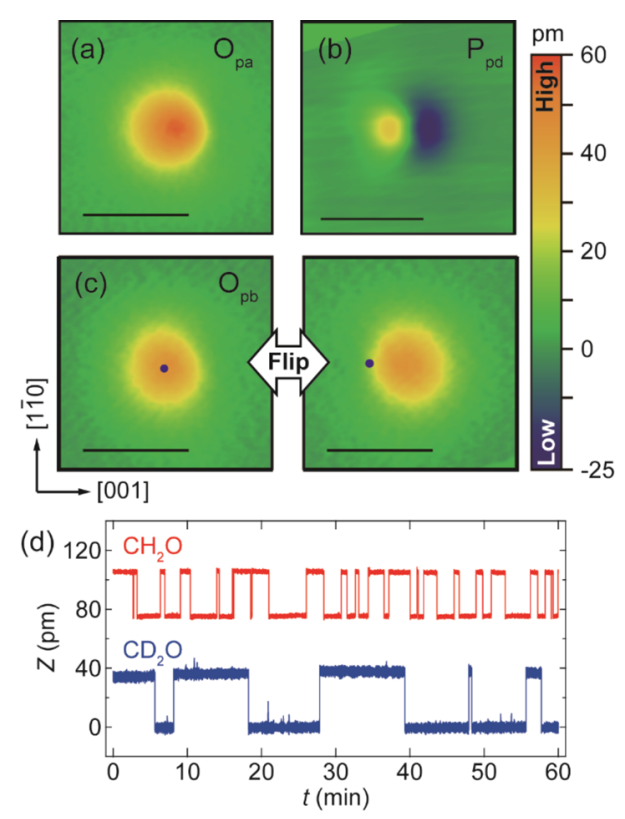

Figure 1. (a)-(c) STM images of three different products obtained by injecting tunneling electrons of $1 \mathrm{~V}$ to methoxy, (a) $\mathrm{O}_{\mathrm{pa}}$ (b) $\mathrm{P}_{\mathrm{pd}}$, and $(\mathrm{c}) \mathrm{O}_{\mathrm{pb}}$, with two mirror-reflected configurations. $\left(4.5 \mathrm{~K}, V_{\text {bias }}=\right.$ $\left.10 \mathrm{mV}, I_{\mathrm{t}}=10 \mathrm{pA}\right) . \mathrm{P}_{\mathrm{pd}}$ and $\mathrm{O}_{\mathrm{pb}}$ have been identified as formaldehyde. $^{27}$ The scale bar in each figure is $1 \mathrm{~nm}$. (d) Traces of the tip height $(\mathrm{Z})$ measured for $\mathrm{CH}_{2} \mathrm{O}$ (red) and $\mathrm{CD}_{2} \mathrm{O}$ (blue). The lateral tip position during the measurement is indicated in (c) with blue dots. The data for $\mathrm{CH}_{2} \mathrm{O}$ are vertically shifted for clarity. The $Z$ traces were acquired at $V_{\text {bias }}=10 \mathrm{mV}$ and $I_{\mathrm{t}}=10 \mathrm{pA}$.

symmetry states spontaneously even at $4.5 \mathrm{~K}$ and a low $V_{\text {bias }}$ like $10 \mathrm{mV}$ (Figure 1c), whereas no flipping was observed for $\mathrm{O}_{\mathrm{pa}}$ and $\mathrm{P}_{\mathrm{pd}}$ at very low $V_{\text {bias }} \mathrm{O}_{\mathrm{pb}}$ and $\mathrm{P}_{\mathrm{pd}}$ have also been reported previously and are identified as formaldehyde. ${ }^{27}$ However, the previous study did not show the flipping of $\mathrm{O}_{\mathrm{pb}}$ below $V_{\text {bias }}$ of $30 \mathrm{mV}$. Here we focus on the flipping of $\mathrm{O}_{\mathrm{pb}}$ at $V_{\text {bias }}=10 \mathrm{mV}$, where vibrational excitation by tunneling electrons is almost negligible.
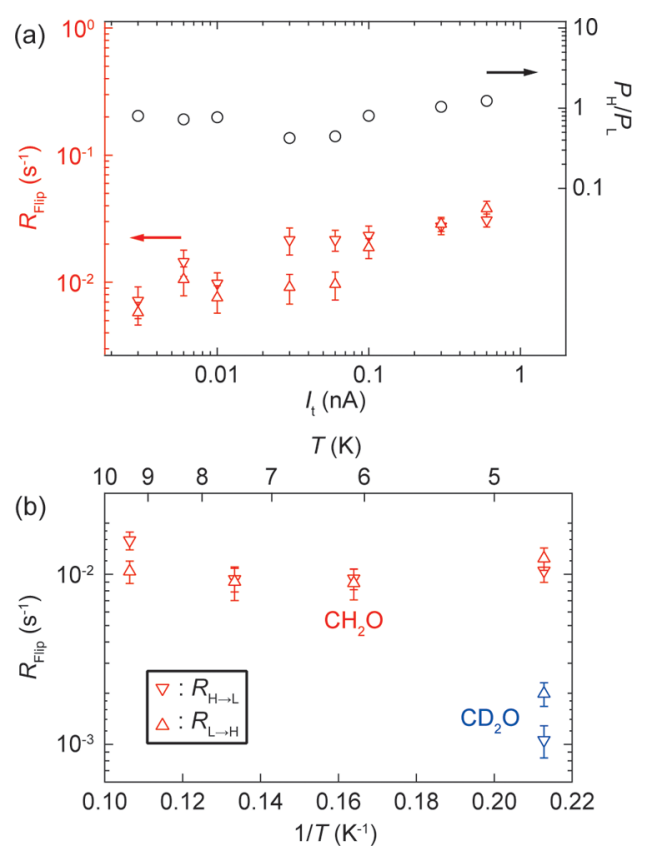

Figure 2. (a) Current dependence of $R_{\text {Flip }}$ for the "high" to "low" transition $\left(R_{\mathrm{H} \rightarrow \mathrm{L}}\right.$, inverted triangle $)$ and the reverse process $\left(R_{\mathrm{L} \rightarrow \mathrm{H}}\right.$, triangle) for $\mathrm{CH}_{2} \mathrm{O}$ acquired at $4.5 \mathrm{~K} . V_{\text {bias }}$ was fixed at $10 \mathrm{mV}$ during the measurement. The black circles in the top part show the current dependence of the fractional population ratio $\left(P_{\mathrm{H}} / P_{\mathrm{L}}\right)$. (b) Temperature dependence of $R_{\text {Flip }}$ for $\mathrm{CH}_{2} \mathrm{O}$ (red). The rate for $\mathrm{CD}_{2} \mathrm{O}$ (blue) at $4.5 \mathrm{~K}$ is plotted for comparison. (a)
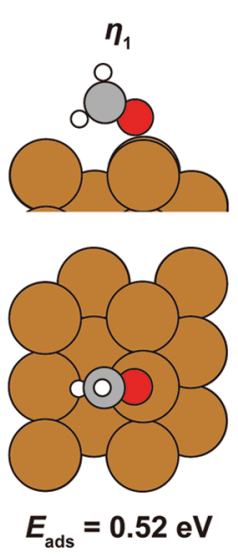

(d)

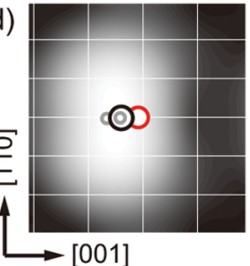

(b)
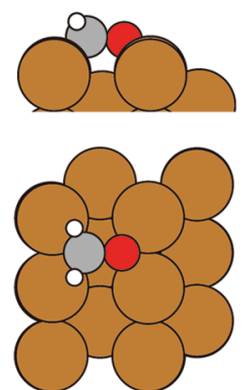

$E_{\text {ads }}=0.87 \mathrm{eV}$

(e)

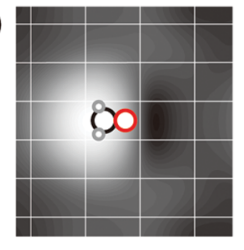

(c)
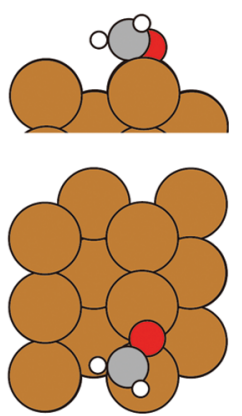

$E_{\text {ads }}=0.64 \mathrm{eV}$

(f)

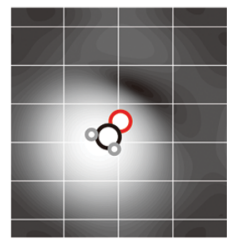

Figure 3. Calculated structures of (a) $\eta^{1}$-, (b) $\eta^{2}$-, and (c) $\eta^{3}$ configurations of the formaldehyde on the $\mathrm{Cu}(110)$ surface. The adsorption energies $\left(E_{\text {ads }}\right)$ are given in the figure. (d) - (f) Simulated topographical STM images at an average tip-surface distances of (a) 10.0 , (b) 7.5 , and (c) $8.7 \AA$ and at the Fermi level with a broadening of $0.1 \mathrm{eV}$. The white grid lines represent the surface lattice of $\mathrm{Cu}(110)$. The structure of the adsorbed formaldehyde is superimposed for each structure.

The flipping can be monitored over the molecule with the lateral tip position fixed at the highest position in the STM 

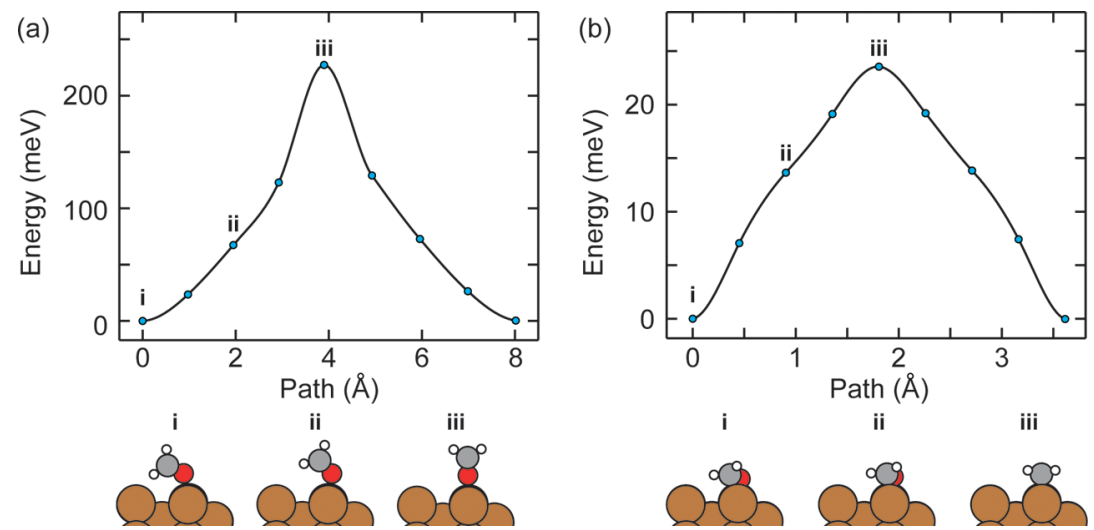

Figure 4. MEP of the flipping reactions of $\eta^{1}$ - and $\eta^{3}$-configurations on the $\mathrm{Cu}(110)$ surface. The path lengths were obtained from the distances between the images in configuration space. The structures along each path are shown in the bottom panel.

Table 1. MEP Effective Barriers Heights $\left(D_{\text {eff }}\right)$ and Lengths $(\Delta s)$ between the Two Mirror-Reflected Configurations, Estimated Tunneling Rates $\left(R_{t}\right)$, and Probabilities $\left(P_{0}\right)$ of the Flipping Reactions of $\eta^{1}$ - and $\eta^{3}$-Configurations on the $\mathrm{Cu}(110)$ Surface $^{a}$

$\begin{array}{ccccc} & D_{\text {eff }}(\mathrm{meV}) & \begin{array}{c}\Delta s \\ (\AA)\end{array} & R_{\mathrm{t}}\left(\mathrm{s}^{-1}\right) & P_{0} \\ \eta^{1} & 214(215) & 7.8 & 2 \times 10^{-56}\left(2 \times 10^{-63}\right) & 6 \times 10^{-70}\left(5 \times 10^{-77}\right) \\ \eta^{3} & 20(21) & 3.62 & 6 \times 10^{-2}\left(1.5 \times 10^{-3}\right) & 3 \times 10^{-14}\left(8 \times 10^{-16}\right)\end{array}$

${ }^{a}$ The values in the parentheses correspond to the ones for the deuterated species $\left(\mathrm{CD}_{2} \mathrm{O}\right)$. The calculations are detailed in the Supporting Information.

image (as indicated by the dots in Figure 1c). The flipping under the tip causes a change in the tip height $(Z)$ through the feedback loop. In the $Z$ trace (as a function of time, $t$ ), a random telegraph signal between two states is observed (Figure 1d), where the "high" and "low" states correspond to the formaldehyde under and away from the tip, respectively. In Figure $1 \mathrm{~d}$, the $Z$ trace measured for normal and deuterated formaldehyde $\left(\mathrm{CD}_{2} \mathrm{O}\right)$ is shown. It is clear that $\mathrm{CH}_{2} \mathrm{O}$ flips much more frequently than $\mathrm{CD}_{2} \mathrm{O}$, indicating a significant KIE. In order to discuss the reaction in a quantitative manner, the flipping rate from the "high" to "low" state $\left(R_{\mathrm{H} \rightarrow \mathrm{L}}\right)$ and the opposite process $\left(R_{\mathrm{L} \rightarrow \mathrm{H}}\right)$ was extracted from the $Z$ trace. Two different methods were used to obtain the rate, by fitting the histogram of the time intervals of the reaction to an exponential decay function or by dividing the total number of reaction events by the total measurement time (both methods are statistically equal). We used the latter method for a very low reaction rate $\left(<1 \times 10^{-3} \mathrm{~s}^{-1}\right)$ since the sampling number of the total reaction events was too limited to apply the histogram analysis.

Figure 2a shows the current dependence of the flipping rate $\left(R_{\text {Flip }}\right)$ for $\mathrm{CH}_{2} \mathrm{O}$ obtained at $V_{\text {bias }}=10 \mathrm{mV}$. We found that the rate depends very weakly on the current. The reaction rate of an adsorbate follows a power law $\left(R \propto I_{t}^{N}\right)$ when induced by tunneling electrons. ${ }^{35}$ Hence, our result indicates that the flipping cannot be ascribed to an electron-induced process. The population ratio of the "high" and "low" states $\left(P_{\mathrm{H}}\right.$ and $P_{\mathrm{L}}$ ) is almost unity (top part of Figure 2a), indicating that the potential energy surface (PES) of the flipping reaction was not significantly distorted by any tip-molecule interaction. The deviation of $P_{\mathrm{H}} / P_{\mathrm{L}}$ from unity at a higher current can be explained by a tip-induced modification of the PES. ${ }^{26}$ It was also found that $R_{\text {Flip }}$ is somewhat affected by the tip conditions (see Supporting Information).

Figure $2 \mathrm{~b}$ shows the temperature dependence of $R_{\text {Flip }}$. If the reaction is thermally activated, a linear relationship with a negative slope is expected between $\log R_{\text {Flip }}$ and $1 / T$. However, $R_{\text {Flip }}$ weakly depends on the temperature below $10 \mathrm{~K}$, indicating that the reaction is governed by tunneling and no thermally assisted process (coupling with different vibrational modes) is involved. ${ }^{36}$ In addition, $R_{\text {Flip }}$ of $\mathrm{CD}_{2} \mathrm{O}$ reveals the KIE of about 1 order of magnitude.

The possible adsorption structures of the formaldehyde and reaction pathways were examined using DFT calculations. Parts $\mathrm{a}-\mathrm{c}$ of Figure 3 summarize the various calculated adsorption geometries of $\mathrm{CH}_{2} \mathrm{O}$ on the $\mathrm{Cu}(110)$ surface. The $\eta_{2}$-configurations are consistent with the structure reported in the previous calculation, ${ }^{37}$ but our calculation reveals that $\eta_{1}$ is adsorbed atop rather than on short bridge. Our simulations found an additional adsorption geometry, denoted as $\eta^{3}$, which breaks all the symmetries of the $\mathrm{Cu}(110)$ surface. Among three adsorption states, $\eta^{2}$ is most stable with an adsorption energy of $0.87 \mathrm{eV}$. The other two adsorption geometries are local energy minimum structures that should also be considered here because they could be kinetically stabilized at cryogenic temperatures. Comparing the calculated STM image in Figure $3 \mathrm{e}$ with the experimental image in Figure $1 \mathrm{~b}, \eta^{2}$ can be assigned to $\mathrm{P}_{\mathrm{pd}}$. The relatively large adsorption energy of $\eta^{2}$ is also consistent with the fact that no flipping occurs in $\mathrm{P}_{\mathrm{pd}}$. However, $\mathrm{O}_{\mathrm{pa}}$ and $\mathrm{O}_{\mathrm{pb}}$ cannot be straightforwardly assigned from the STM images, but we were able to identify these two species from the analysis of the flipping mechanism of the $\eta^{1}$ and $\eta^{3}$-configurations as detailed below.

We examined the minimum energy path (MEP) of the flipping reaction using the nudged elastic band method. Figure 4 shows the MEP for $\eta^{1}$ - and $\eta^{3}$-configurations between the two mirror-reflected structures. The MEP for $\eta^{1}$ (Figure 4a) was constrained to be in the symmetry plane spanned by the surface normal and the [001] direction and the reaction occurs by a vertical wagging motion. The saddle point of this MEP has two imaginary frequencies. The MEP for $\eta^{3}$ corresponds to an azimuthal rotation of the $\mathrm{CH}_{2}$ group around the surface normal (Figure $4 \mathrm{~b}$ ). The saddle point of this MEP has a single imaginary frequency and corresponds to a transition structure. The potential energy barrier for $\eta^{3}$ is only $\sim 24 \mathrm{meV}$ and is $\sim 10$ times smaller than the one for $\eta^{1}$. The effective barrier becomes $\sim 20$ and $\sim 21 \mathrm{meV}$ for $\mathrm{CH}_{2} \mathrm{O}$ and $\mathrm{CD}_{2} \mathrm{O}$, respectively, when the contribution of the zero-point energy 
of the vibration along the reaction coordinate is included (see Supporting Information for the details). This very small barrier allows for carbon tunneling in the flipping reaction. At the experimental temperature of $4.5 \mathrm{~K}$, no vibrational modes are thermally populated, since all vibrational modes have energies larger than $\sim 7 \mathrm{meV}$. As shown in Table 1, a simple but rough estimate of the tunneling probability based on the calculated MEPs with the WKB approximation (see Supporting Information for the details) shows that $\eta^{3}$ can be flipped by tunneling through the small barrier, whereas the tunneling process is completely negligible for $\eta^{1}$. On the basis of the above findings, we assign our tunneling species $\mathrm{O}_{\mathrm{pb}}$ to $\eta^{3}$ and $\mathrm{O}_{\mathrm{pa}}$ to $\eta^{1}$. In the previously observed carbon tunneling reactions, the displacement of the carbon atom was extremely small $(\sim 0.1 \AA),{ }^{38}$ thus resulting in a very narrow barrier that allows tunneling. In the flipping of formaldehyde, the carbon displacement of $\sim 1.1 \AA$ between the two mirror-reflected configurations is substantially longer but has a very small potential barrier, representing another condition for carbon tunneling. The difference in the tunneling rates between $\mathrm{CH}_{2} \mathrm{O}$ and $\mathrm{CD}_{2} \mathrm{O}$ is caused predominantly by the mass difference, since the differences in the effective barrier heights are small. In the WKB approximation, the estimated tunneling rates for $\mathrm{CH}_{2} \mathrm{O}$ and $\mathrm{CD}_{2} \mathrm{O}$ give a $\mathrm{KIE}$ of $\sim 40$, which is in reasonable agreement considering the simplicity of this approximation and the limited accuracy of DFT calculation to describe potential energy barriers.

In summary, we identified three different adsorption configurations of formaldehyde on the $\mathrm{Cu}(110)$ surface by combined STM experiments and DFT calculations. Remarkably, it was found that the flipping reaction of the $\eta_{3^{-}}$ configuration occurs by tunneling including the carbon atom, which is evident from the temperature-independent reaction rate below $10 \mathrm{~K}$. The MEP analysis revealed that the $\eta_{3^{-}}$ configuration exhibits a very small barrier of $\sim 20(\sim 21) \mathrm{meV}$ for the flipping reaction of $\mathrm{CH}_{2} \mathrm{O}\left(\mathrm{CD}_{2} \mathrm{O}\right)$, which allows for carbon tunneling between the vibrational ground states of the mirror-reflected configuration.

\section{ASSOCIATED CONTENT}

\section{S Supporting Information}

The Supporting Information is available free of charge on the ACS Publications website at DOI: 10.1021/acs.jpclett.8b03806.

Computational details, estimate of tunneling rate, and influence of the tip conditions on the flipping rate (PDF)

\section{AUTHOR INFORMATION}

\section{Corresponding Author}

*E-mail: kuma@fhi-berlin.mpg.de.

\section{ORCID}

Mariana Rossi: 0000-0002-3552-0677

Takashi Kumagai: 0000-0001-7029-062X

\section{Notes}

The authors declare no competing financial interest.

\section{ACKNOWLEDGMENTS}

The authors acknowledge $H$. Okuyama for valuable discussions. T.K. acknowledges the support by JST-PRESTO (JPMJPR16S6). E.D. and M.P. acknowledge computer time allocated on THOMAS at the MMM hub partially funded by EPSRC (EP/L000202, EP/P020194) through the Materials Chemistry Consortium.

\section{REFERENCES}

(1) Bell, R. P. The Application of Quantum Mechanics to Chemical Kinetics. Proc. R. Soc. London, Ser. A 1933, 139, 466-474.

(2) Bell, R. The Tunneling Effect in Chemistry; Chapman and Hall: London and New York, 1980.

(3) Hynes, J. T.; Klinman, J. P.; Limbach, H.-H.; Schowen, R. L. Hydrogen-Transfer Reactions; Wiley-VCH, 2007.

(4) More O'Ferrall, R. A. Special Issue: Symposium in Print on Tunnelling in Chemical and Biological Reactions. J. Phys. Org. Chem. 2010, 23, 559.

(5) Klinman, J. P.; Kohen, A. Hydrogen Tunneling Links Protein Dynamics to Enzyme Catalysis. Annu. Rev. Biochem. 2013, 82, 471496.

(6) Carpenter, B. K. Heavy-atom Tunneling as the Dominant Pathway in a Solution-phase Reaction? Bond Shift in Antiaromatic Annulenes. J. Am. Chem. Soc. 1983, 105, 1700-1701.

(7) Orendt, A. M.; Arnold, B. R.; Radziszewski, J. G.; Facelli, J. C.; Malsch, K. D.; Strub, H.; Grant, D. M.; Michl, J. Carbon-13 NMR and Polarized IR Spectra of Vicinally Labeled Cyclobutadiene- ${ }^{13} \mathrm{C}_{2}$ in an Argon Matrix: Interconversion of Valence Tautomers. J. Am. Chem. Soc. 1988, 110, 2648-2650.

(8) Lefebvre, R.; Moiseyev, N. Automerization of Cyclobutadiene. J. Am. Chem. Soc. 1990, 112, 5052-5054.

(9) Zuev, P. S.; Sheridan, R. S.; Albu, T. V.; Truhlar, D. G.; Hrovat, D. A.; Borden, W. T. Carbon Tunneling from a Single Quantum State. Science 2003, 299, 867-870.

(10) Sekiya, H.; Nagashima, Y.; Tsuji, T.; Nishimura, Y.; Mori, A.; Takeshita, H. Vibrational Mode-specific Tunneling Splittings in the A States of Deuterated Tropolones. J. Phys. Chem. 1991, 95, 1031110317.

(11) Redington, R. L.; Redington, T. E.; Blake, T. A.; Sams, R. L.; Johnson, T. J. ${ }^{18} \mathrm{O}$ Effects on the Infrared Spectrum and Skeletal Tunneling of Tropolone. J. Chem. Phys. 2005, 122, 224311.

(12) Inui, H.; Sawada, K.; Oishi, S.; Ushida, K.; McMahon, R. J. Aryl Nitrene Rearrangements: Spectroscopic Observation of a Benzazirine and Its Ring Expansion to a Ketenimine by Heavy-atom Tunneling. J. Am. Chem. Soc. 2013, 135, 10246-10249.

(13) Ertelt, M.; Hrovat, D. A.; Borden, W. T.; Sander, W. HeavyAtom Tunneling in the Ring Opening of a Strained Cyclopropene at Very Low Temperatures. Chem. - Eur. J. 2014, 20, 4713-4720.

(14) Schleif, T.; Mieres-Perez, J.; Henkel, S.; Ertelt, M.; Borden, W. T.; Sander, W. The Cope Rearrangement of 1,5-Dimethylsemibullvalene-2(4) $-d_{1}$ : Experimental Evidence for Heavy-atom Tunneling. Angew. Chem., Int. Ed. 2017, 56, 10746-10749.

(15) Vetticatt, M. J.; Singleton, D. A. Isotope Effects and Heavyatom Tunneling in the Roush Allylboration of Aldehydes. Org. Lett. 2012, 14, 2370-2373.

(16) Doubleday, C.; Armas, R.; Walker, D.; Cosgriff, C. V.; Greer, E. M. Heavy-Atom Tunneling Calculations in Thirteen Organic Reactions: Tunneling Contributions are Substantial, and Bell's Formula Closely Approximates Multidimensional Tunneling at $\geq$ 250 K. Angew. Chem., Int. Ed. 2017, 56, 13099-13102.

(17) Lauhon, L. J.; Ho, W. Direct Observation of the Quantum Tunneling of Single Hydrogen Atoms with a Scanning Tunneling Microscope. Phys. Rev. Lett. 2000, 85, 4566-4569.

(18) Kumagai, T.; Kaizu, M.; Hatta, S.; Okuyama, H.; Aruga, T.; Hamada, I.; Morikawa, Y. Direct Observation of Hydrogen-Bond Exchange within a Single Water Dimer. Phys. Rev. Lett. 2008, 100, 166101-166104.

(19) Meng, X.; Guo, J.; Peng, J.; Chen, J.; Wang, Z.; Shi, J.-R.; Li, X.Z.; Wang, E.-G.; Jiang, Y. Direct Visualization of Concerted Proton Tunnelling in a Water Nanocluster. Nat. Phys. 2015, 11, 235-239.

(20) Koch, M.; Pagan, M.; Persson, M.; Gawinkowski, S.; Waluk, J.; Kumagai, T. Direct Observation of Double Hydrogen Transfer via 
Quantum Tunneling in a Single Porphycene Molecule on a $\mathrm{Ag}(110)$ Surface. J. Am. Chem. Soc. 2017, 139, 12681-12687.

(21) Kumagai, T.; Ladenthin, J. N.; Litman, Y.; Rossi, M.; Grill, L.; Gawinkowski, S.; Waluk, J.; Persson, M. Quantum Tunneling in Real Space: Tautomerization of Single Porphycene Molecules on the (111) Surface of $\mathrm{Cu}, \mathrm{Ag}$, and Au. J. Chem. Phys. 2018, 148, 102330.

(22) Heinrich, A. J.; Lutz, C. P.; Gupta, J. A.; Eigler, D. M. Molecule Cascades. Science 2002, 298, 1381-1387.

(23) Repp, J.; Meyer, G.; Rieder, K.-H.; Hyldgaard, P. Site Determination and Thermally Assisted Tunneling in Homogenous Nucleation. Phys. Rev. Lett. 2003, 91, 206102.

(24) Stroscio, J. A.; Celotta, R. J. Controlling the Dynamics of a Single Atom in Lateral Atom Manipulation. Science 2004, 306, 242247.

(25) Nacci, C.; Fölsch, S.; Zenichowski, K.; Dokić, J.; Klamroth, T.; Saalfrank, P. Current versus Temperature-Induced Switching in a Single-Molecule Tunnel Junction: 1,5 Cyclooctadiene on Si(001). Nano Lett. 2009, 9, 2996-3000.

(26) Ladenthin, J. N.; Frederiksen, T.; Persson, M.; Sharp, J. C.; Gawinkowski, S.; Waluk, J.; Kumagai, T. Force-induced Tautomerization in a Single Molecule. Nat. Chem. 2016, 8, 935-940.

(27) Kitaguchi, Y.; Shiotari, A.; Okuyama, H.; Hatta, S.; Aruga, T. Imaging Sequential Dehydrogenation of Methanol on $\mathrm{Cu}(110)$ with a Scanning Tunneling Microscope. J. Chem. Phys. 2011, 134, 174703.

(28) Kresse, G.; Furthmüller, J. Efficient Iterative Schemes for ab initio Total-energy Calculations using a Plane-wave Basis Set. Phys. Rev. B: Condens. Matter Mater. Phys. 1996, 54, 11169-11186.

(29) Kresse, G.; Joubert, D. From Ultrasoft Pseudopotentials to the Projector Augmented-wave Method. Phys. Rev. B: Condens. Matter Mater. Phys. 1999, 59, 1758-1775.

(30) Dion, M.; Rydberg, H.; Schröder, E.; Langreth, D. C.; Lundquist, B. I. Van der Waals Density Functional for General Geometries. Phys. Rev. Lett. 2004, 92, 246401-246404.

(31) Román-Pérez, G.; Soler, J. M. Efficient Implementation of a van der Waals Density Functional: Application to Double-Wall Carbon Nanotubes. Phys. Rev. Lett. 2009, 103, 096102.

(32) Berland, K.; Hyldgaard, P. Exchange Functional that Tests the Robustness of the Plasmon Description of the Van der Waals Density Functional. Phys. Rev. B: Condens. Matter Mater. Phys. 2014, 89, 035412.

(33) Mills, G.; Jónsson, H.; Schenter, G. K. Reversible Work Transition State Theory: Application to Dissociative Adsorption of Hydrogen. Surf. Sci. 1995, 324, 305-337.

(34) Henkelman, G.; Uberuaga, B. P.; Jónsson, H. A. A Climbing Image Nudged Elastic Band Method for Finding Saddle Points and Minimum Energy Paths. J. Chem. Phys. 2000, 113, 9901-9904.

(35) Stipe, B. C.; Rezaei, M. A.; Ho, W.; Gao, S.; Persson, M.; Lundqvist, B. I. Single-Molecule Dissociation by Tunneling Electrons. Phys. Rev. Lett. 1997, 78, 4410-4413.

(36) Litman, Y.; Richardson, J. O.; Kumagai, T.; Rossi, M. Elucidating the Quantum Dynamics of Intramolecular Double Hydrogen Transfer in Porphycene. J. Am. Chem. Soc. 2019, DOI: $10.1021 /$ jacs.8b12471.

(37) Sakong, S.; Groß, A. Density Functional Theory Study of the Partial Oxidation of Methanol on Copper Surfaces. J. Catal. 2005, 231, 420-429.

(38) Borden, W. T. Reactions That Involve Tunneling by Carbon and the Role That Calculations Have Played in Their Study. WIREs Comput. Mol. Sci. 2016, 6, 20-46. 